\title{
Acinetobacter species meningitis in children: a case series from Karachi, Pakistan
}

\author{
Ali Faisal Saleem, Muhammad Shafaat Shah, Abdul Sattar Shaikh, Fatima Mir, Anita K M Zaidi \\ Department of Pediatrics and Child Health, Aga Khan University, Karachi, Pakistan
}

\begin{abstract}
Introduction: Multidrug-resistant strains of Acinetobacter pose a serious therapeutic dilemma in hospital practice, particularly when they cause meningitis, as the few antimicrobial agents to which these isolates are susceptible have poor central nervous system (CNS) penetration. Methodology: We retrospectively reviewed the clinical course and outcome of eight consecutive cases of meningitis due to Acinetobacter spp. in children ages 15 years or less, seen in a tertiary care medical center in Karachi, Pakistan.

Results: Of the eight cases of Acinetobacter meningitis, isolates from five patients were pan-resistant, and two were multidrug-resistant. A neurosurgical procedure was performed in five of eight patients followed by external ventricular drain insertion prior to the development of infection. Seven received intravenous (IV) polymyxin (mean; 12.8 days), while 5/8 also received intrathecal (IT) polymyxin (mean; 12.0 days). The mean length of hospitalization was $38.7 \pm 19$ days. All patients achieved cerebrospinal fluid (CSF) culture negativity by the end of treatment (mean; 5.4 days). Two patients died: one with pan-resistant Acinetobacter, and the second with a multi-drug resistant isolate.

Conclusion: Post-neurosurgical multidrug-resistant and pan-resistant Acinetobacter meningitis can be successfully treated if appropriate antimicrobial therapy is instituted early. The role of IT polymyxin B administration alone versus combination therapy (IV and IT) needs further study.
\end{abstract}

Key words: multidrug-resistant Acinetobacter meningitis; mortality; children; neurosurgical procedure

J Infect Dev Ctries 2011; 5(11):809-814.

(Received 28 October 2010 - Accepted 02 June 2011)

Copyright (C) 2011 Saleem et al. This is an open-access article distributed under the Creative Commons Attribution License, which permits unrestricted use, distribution, and reproduction in any medium, provided the original work is properly cited.

\section{Introduction}

Acinetobacter baumannii is a nosocomial pathogen that threatens health-care systems worldwide, resulting in high morbidity and mortality in critically ill patients [1,2]. Although nosocomial pneumonia is the most common clinical manifestation, Acinetobacter meningitis has been increasing in frequency [3]. Acinetobacter meningitis may occur following neurosurgery, traumatic head injury or/and metastatic infection in bacteremic patients. Multidrug-resistant Acinetobacter (MDRA) is defined as resistance to more than two of the five classes of drugs: cephalosporins, carbapenems, ampicillin-sulbactam, fluoroquinolones and aminoglycosides [1,4]. Pan-resistant Acinetobacter (PRA) is defined as resistance to all five classes of the drugs mentioned above $[1,4]$. For PRA infections in resource-limited settings, the only available therapeutic option is polymyxin [5]. Tigecycline, a glycylcycline antibiotic, has good in vitro activity against PRA [6], but is very costly and not readily available in developing countries. In addition, tigecycline has poor penetration to the central nervous system [7].

The mortality rate of central nervous system infection due to MDRA has been reported to range from $15-71 \%$ [3]. There are very few published studies of neurological outcomes in pediatric MDRA meningitis cases $[8,9]$. We report our experience with eight cases of Acinetobacter meningitis in children, seen in a tertiary care medical center in Karachi, Pakistan.

\section{Methodology}

\section{Case inclusion criteria}

Eight consecutive cases of children diagnosed with Acinetobacter meningitis during the period of July 2006 to June 2010 were identified by using the hospital medical records and the health information management system at the Aga Khan University Hospital, Karachi, Pakistan. Inclusion criteria for the study required the isolation of Acinetobacter spp. from cerebrospinal fluid (CSF) of patients less than or equal to 15 years in age. All the cases had been 
Table 1. Clinical profile of patients with Acinetobacter meningitis

\begin{tabular}{|c|c|c|c|c|c|}
\hline Patient & Age/Gender & Risk Factor/s & Neurosurgical procedure & $\operatorname{LOS}^{1}$ & $\begin{array}{c}\text { Discharge } \\
\text { Disposition }\end{array}$ \\
\hline 1 & $9 \mathrm{yr} / \mathrm{F}$ & $\mathrm{NSP}^{2}$ & $\begin{array}{c}\text { Posterior fossa craniotomy \& } \\
\text { excision of tumor } \\
\text { EVD }^{3} \text { insertion } \\
\text { VPS insertion } \\
\end{array}$ & 49 & Alive \\
\hline 2 & $5 \mathrm{mo} / \mathrm{M}$ & NSP,PMV ${ }^{4}$ & EVD insertion & 43 & Died \\
\hline 3 & $9 \mathrm{yr} 1 \mathrm{mo} / \mathrm{M}$ & NSP & $\begin{array}{l}\text { Left frontal craniotomy \& } \\
\text { excision of tumor } \\
\text { EVD insertion } \\
\text { EVD re- insertion } \\
\text { VPS }^{5} \text { insertion }\end{array}$ & 56 & Alive \\
\hline 4 & 12 yr $9 \mathrm{mo} / \mathrm{M}$ & $\begin{array}{l}\text { Road traffic } \\
\text { injury, NSP, } \\
\text { severe } \\
\text { malnutrition }\end{array}$ & $\begin{array}{c}\text { Bifrontal craniotomy, } \\
\text { evacuation of hematoma } \& \\
\text { duraplasty } \\
\text { EVD insertion }\end{array}$ & 61 & Died \\
\hline 5 & $9 \mathrm{mo} / \mathrm{M}$ & $\begin{array}{l}\text { NSP, severe } \\
\text { malnutrition }\end{array}$ & $\begin{array}{l}\text { EVD insertion } \\
\text { VPS placement }\end{array}$ & 21 & Alive \\
\hline 6 & 5 days/M & PMV & None & 58 & Alive \\
\hline 7 & $3 \mathrm{mo} / \mathrm{F}$ & NSP, PMV & EVD insertion & 11 & Alive \\
\hline 8 & $2 \mathrm{mo} / \mathrm{F}$ & NSP & EVD insertion & 11 & Alive \\
\hline
\end{tabular}

${ }^{1}$ LOS (length of stay (days); ${ }^{2} \mathrm{NSP}$ (neurosurgical procedure); ${ }^{3} \mathrm{PMV}$ (prolonged mechanical ventilation); ${ }^{4} \mathrm{EVD}$ (external ventricular drain);

${ }^{5}$ VPS (ventriculo-peritoneal shunt)

located in either the pediatric intensive care unit (PICU) or the neonatal intensive care unit (NICU) of Aga Khan University Hospital.

Demographic characteristics included age, gender, anthropometry (weight, length or height measured at the time of admission), and discharge disposition (dead, alive). Information regarding length of hospital stay (LOS) and duration and mode of treatment administration was also collected. In addition, known risk factors for Acinetobacter meningitis, such as surgical procedures, head injury and isolation of Acinetobacter spp. from sites other than CSF were recorded.

The diagnosis of Acinetobacter meningitis was based on Centers for Disease Control (CDC) criteria [10], which calls for the positive identification of CSF Acinetobacter culture and the presence of at least one feature from each of the following criteria, indicating the presence of meningitis: a) clinical presentation with acute onset of fever $\left(>38^{\circ} \mathrm{C}\right)$ and signs of meningeal irritation; or b) elevated CSF white blood cells and proteins and/or decreased glucose coupled with Acinetobacter isolated from blood $[10,11]$.

\section{Antimicrobial resistance}

The Acinetobacter spp. isolates were divided into the following three drug resistance categories:

(i) Pan-resistant Acinetobacter (PRA) was defined as isolates resistant to all antimicrobial agents considered first-line therapy for Acinetobacter infections. These included anti-pseudomonal cephalosporins (ceftazidime or cefepime), antipseudomonal carbapenems (imipenem or meropenem), ampicillin-sulbactam, fluoroquinolones (ciprofloxacin or levofloxacin), and aminoglycosides (gentamicin, tobramycin or amikacin) $[1,4]$.

(ii) Multidrug-resistant Acinetobacter (MDRA) was defined as strains resistant to more than two of the following five drug classes: anti-pseudomonal cephalosporin (ceftazidime or cefepime), antipseudomonal carbapenems (imipenem or meropenem), ampicillin-sulbactam, fluoroquinolones (ciprofloxacin or levofloxacin) and aminoglycosides (gentamicin, tobramycin or amikacin) $[1,4]$.

(iii) Susceptible Acinetobacter (SA) was defined as susceptible to all first-line drug classes $[1,4]$. 
Table 2. Antimicrobial susceptibility of Acinetobacter from CSF and Blood

\begin{tabular}{|cccc|}
\hline Patient & Isolate Site $^{\mathbf{1}}$ & $\begin{array}{c}\text { Antibiotic Resistance } \\
\text { Category }\end{array}$ & Susceptibility Pattern \\
\cline { 2 - 5 } & $\begin{array}{c}\text { CSF (8), Blood } \\
(11)\end{array}$ & PRA $^{2}$ & polymyxin \\
\hline 2 & CSF (32) & PRA & polymyxin, tobramycin \\
\hline 3 & CSF (43) & PRA & polymyxin, amikacin, meropenem, imipenem \\
\hline 4 & $\begin{array}{c}\text { Blood (2), CSF } \\
(10)\end{array}$ & MDRA $^{3}$ & polymyxin \\
\hline 5 & CSF (14) & PRA & polymyxin, tobramycin \\
\hline 6 & $\begin{array}{c}\text { Blood(16),CSF } \\
(32)\end{array}$ & PRA & polymyxin, tobramycin, gentamicin, tetracycline \\
\hline 7 & $\begin{array}{c}\text { CSF (8); Blood } \\
(11)\end{array}$ & MDRA $^{\text {MSA }}$ & $\begin{array}{c}\text { polymyxin, amikacin, meropenem, imipenem, gentamicin, } \\
\text { ceftriaxone }\end{array}$ \\
\hline 8 & CSF (3) & SA $^{4}$ &
\end{tabular}

${ }^{1}$ Bracket shows the day of hospitalization on which pathogen isolated; ${ }^{2}$ Pan- resistant Acinetobacter, ${ }^{3}$ Multidrug resistant Acinetobacter, ${ }^{4}$ Susceptible Acinetobacter

Table 3. Therapeutic administration and time to culture negativity

\begin{tabular}{|c|c|c|c|c|c|c|c|}
\hline \multirow{2}{*}{ Patient } & \multicolumn{3}{|c|}{ Intravenous therapy } & \multicolumn{3}{|c|}{ Intrathecal therapy } & \multirow{2}{*}{$\begin{array}{c}\text { Time to } \\
\text { Negative } \\
\text { CSF in Days }\end{array}$} \\
\hline & Drug & Dosage/Day & \# Days & Drug & Dosage & \# Days & \\
\hline 1 & polymyxin & $40,000 \mathrm{IU} / \mathrm{kg}$ & 19 & polymyxin & $2000 \mathrm{IU} / \mathrm{kg}$ & 21 & 7 \\
\hline 2 & polymyxin & $40,000 \mathrm{IU} / \mathrm{kg}$ & 24 & polymyxin & $3000 \mathrm{IU} / \mathrm{kg}$ & 10 & 4 \\
\hline 3 & polymyxin & $40,000 \mathrm{IU} / \mathrm{kg}$ & 6 & polymyxin & $2000 \mathrm{IU} / \mathrm{kg}$ & 5 & 6 \\
\hline 4 & polymyxin & $40,000 \mathrm{IU} / \mathrm{kg}$ & 10 & amikacin & $7.5 \mathrm{mg} / \mathrm{kg}$ & 7 & 7 \\
\hline 5 & polymyxin & $50,000 \mathrm{IU} / \mathrm{kg}$ & 6 & polymyxin & $2500 \mathrm{IU} / \mathrm{kg}$ & 5 & 5 \\
\hline 6 & polymyxin & $40,000 \mathrm{IU} / \mathrm{kg}$ & 16 & none & & & 7 \\
\hline 7 & polymyxin & $40,000 \mathrm{IU} / \mathrm{kg}$ & 11 & polymyxin & $5000 \mathrm{IU} / \mathrm{kg}$ & 8 & 3 \\
\hline 8 & $\begin{array}{l}\text { ceftriaxone } \\
\text { amikacin }\end{array}$ & $\begin{array}{c}100 \mathrm{mg} / \mathrm{kg} \\
15 \mathrm{mg} / \mathrm{kg}\end{array}$ & $\begin{array}{l}14 \\
14\end{array}$ & none & & & 4 \\
\hline
\end{tabular}

\section{Organism identification and antimicrobial susceptibility}

Acinetobacter spp. identification was determined by Gram stain, colony morphology and conventional biochemical tests such as oxidase, sulfide-indolemotility, citrate utilization, urea hydrolysis and triple sugar iron agar tests. API 20NE (bioMérieux, Marcy l'Etoile, France) was utilized when the above tests were inconclusive. Acinetobacter is endemic in the institution and prior surveillance data demonstrated the predominant species as Acinetobacter baumannii; hence identification to the species level was not routinely performed. The Clinical and Laboratory Standards Institute (CLSI) guidelines for antimicrobial susceptibility testing were utilized for the performance and determination of disk diffusion interpretive criteria for antibiotics tested. Polymyxin discs of 300 units were used for susceptibility testing $[1,12,13]$. The disc diffusion technique was reported to be an unreliable method for evaluating the susceptibility of bacteria to polymyxins because these antibiotics diffuse poorly in agar. Consequently results of polymyxin disk diffusion tests should be confirmed with a broth dilution method $[14,15]$.

\section{Results}

The mean age of the eight patients in this study was 48.7 months (range 5 days to 12.9 years); five were infants. Table 1 displays the age, gender, risk factors, length of hospital stay (LOS) and patient 
outcome. Two patients, aged 9 months and 12.9 years, had severe malnutrition as an additional risk factor for nosocomial infection. Neurosurgical procedures were performed in five patients $(62.5 \%)$ prior to developing Acinetobacter meningitis and in two of these CSF cultures became positive within 48 hours of the procedure. External ventricular drains (EVD) were placed in seven patients $(87.5 \%)$. Five of eight patients were infected with PRA, two with MDRA and one was SA (Table 2). Blood cultures from four patients (50\%) grew Acinetobacter and the isolate concurrently caused respiratory infections in two cases and a wound infection in another. In Table 3 the antibiotic dosage, mode and duration of therapy as well as the time of CSF culture negativity is shown. The mean treatment days for IV and IT polymyxin were 12.8 (range $6-24$ ) days and 12.0 (range $5-21$ ) days, respectively. Mean duration of hospitalization was 38.7 days. In all patients the CSF cultures were negative by the end of treatment. The mean time to CSF culture negativity was 5.4 (range 3 - 7) days. Two patients (2/8) died, one of multiorgan failure with pulmonary hemorrhage and the other of hypoxic brain injury secondary to bifrontal contusion; both had received IV and IT polymyxin.

\section{Discussion}

The success of Acinetobacter as a highly transmissible nosocomial pathogen that causes serious infections such as meningitis, sepsis and pneumonia is well-established [4]. Factors implicated in patient-to-patient transmission and multidrug resistance include widespread use of cephalosporins and suboptimal hand hygiene among health-care workers [16,17]. Meningitis in post-neurosurgical patients poses a serious therapeutic challenge due to limited therapeutic choices [11] and the association with poor clinical outcomes (high mortality and debilitating neurological sequelae) $[12,13]$.

Polymyxins have been re-introduced in the clinical armamentarium for Acinetobacter infections as the drug of last resort, when the pathogen is resistant to other antibiotics. Polymyxins are a group of polypeptide antibiotics that includes five different chemical compounds (polymyxins A through E); however, only polymyxin B and E (colistin) are used in clinical practice [18].Polymyxin B differs from colistin by only one amino acid [19], both of which were found to have similar antibacterial spectra, efficacy and adverse effects in a recent comparative study [20]. Although polymyxins had been discontinued from treatment regimens because of nephro and neuro- toxicity, they are now used to treat pan-resistant pathogens.

Since PRA was first reported from Taiwan in 1998, there has been a global trend of evolution of MDRA to PRA [21]. This increase in pan-resistance has been observed in our medical centre over the past few years as well, and has led to the almost exclusive use of polymyxin as treatment in our patients for Acinetobacter infections [1]. At present, resistance to polymyxin is rare and none was observed in our study cohort $[22,23]$. Pediatric use of polymyxin has recently been reported particularly in post neurosurgical procedure meningitis $[8,24]$; however, long-term outcomes have not been established.

Polymyxins have poor CNS penetration following systemic administration hence necessitating intrathecal delivery of the drug $[18,25]$. The use of intravenous (IV) polymyxins, however, has been reported to successfully cure meningitides due to Pseudomonas aeruginosa and Acinetobacter baumannii in a systemic review [25]. This may be of considerable significance in health-care settings where intrathecal administration is not feasible. Direct administration of colistin into the CSF is successful, well tolerated, and relatively less nephrotoxic than IV drug delivery [18]. Nephrotoxicity (as measured by an increased creatinine clearance - $\mathrm{CrCl}$ ) has been reported in 6$14 \%$ and $10-37 \%$ in patients treated with polymyxin B and colistin, respectively [5]. Oliveira et al. found no difference in adverse effects between the two [20]. We checked $\mathrm{CrCl}$ levels in all patients prior to polymyxin administration. Only one patient required renal adjustment of polymyxin. Dose dependent but reversible neurotoxicity (meningeal irritation, nerve impingement and seizures) has also been reported in patients treated with IT polymyxin [5,25]. None of these adverse events occurred in any of our patients. CSF culture negativity was achieved in all patients, including two who received IV polymyxin alone. Colistin levels have been detected in inflamed meninges [5,26] but limited data show that polymyxin $\mathrm{B}$ penetrates poorly into the CSF, even when the meninges are inflamed.

In our cohort, polymyxin was used for PRA isolates. Although the efficacy of polymyxin administration via two routes (IV and IT) simultaneously remains unclear [25,27], these routes of administration for the treatment of meningitis have been reported [28-30]. Rodriguez et al. reported higher cure rates in those neurosurgical patients who were treated with IV and IT colistin than those who 
were treated with carbapenem alone [28]. No clinical guidelines are available for combination therapy for PRA or MDRA. We did not encounter chemical ventriculitis, associated with intrathecal therapy [3] in our patients.

\section{Conclusion}

MDRA and PRA are associated with high morbidity and mortality in children. Early diagnosis and treatment are crucial. Polymyxin is a safe and effective drug in pediatric patients when administered either IV and/or IT.

\section{Acknowledgements}

Drs. Ali Faisal Saleem and Fatima Mir received research training support from the National Institute of Health's Fogarty International Center (1 D43 TW007585-01).

\section{References}

1. Saleem AF, Ahmed I, Mir F, Ali SR, Zaidi AK (2009) Panresistant Acinetobacter infection in neonates in Karachi, Pakistan. J Infect Dev Ctries 4: 30-37.

2. Talbot GH, Bradley J, Edwards JE Jr., Gilbert D, Scheld M, Bartlett JG (2006) Bad bugs need drugs: an update on the development pipeline from the Antimicrobial Availability Task Force of the Infectious Diseases Society of America. Clin Infect Dis 42: 657-668.

3. Kim BN, Peleg AY, Lodise TP, Lipman J, Li J, Nation R, Paterson DL (2009) Management of meningitis due to antibiotic-resistant Acinetobacter species. Lancet Infect Dis 9: 245-255.

4. Peleg AY, Seifert H, Paterson DL (2008) Acinetobacter baumannii: emergence of a successful pathogen. Clin Microbiol Rev 21: 538-582.

5. Landman D, Georgescu C, Martin DA, Quale J (2008) Polymyxins revisited. Clin Microbiol Rev 21: 449-465.

6. Karageorgopoulos DE, Kelesidis T, Kelesidis I, Falagas ME (2008) Tigecycline for the treatment of multidrug-resistant (including carbapenem-resistant) Acinetobacter infections: a review of the scientific evidence. J Antimicrob Chemother 62: 45-55.

7. Ray L, Levasseur K, Nicolau DP, Scheetz MH (2010) Cerebral spinal fluid penetration of tigecycline in a patient with Acinetobacter baumannii cerebritis. Ann Pharmacother 440: 582-586.

8. Lee SY, Lee JW, Jeong DC, Chung SY, Chung DS, Kang JH (2008) Multidrug-resistant Acinetobacter meningitis in a 3 -year-old boy treated with i.v. colistin. Pediatr Int 50: 584585.

9. Ozaki T, Nishimura N, Arakawa Y, Suzuki M, Narita A, Yamamoto Y, Koyama N, Nakane K, Yasuda N, Funahashi K (2009) Community-acquired Acinetobacter baumannii meningitis in a previously healthy 14-month-old boy. J Infect Chemother 15: 322-324.

10. RP. HTG. Surveillance of nosocomial infections. In: Mayhall CG, editor. Hospital Epidemiology and Infection Control. 3rd ed. Philadelphia: Lipponcott Williames and Wilkings. 2004: 1659-1702.

11. Horan TC, Andrus M, Dudeck MA (2008) CDC/NHSN surveillance definition of health care-associated infection and criteria for specific types of infections in the acute care setting. Am J Infect Control 36: 309-332.

12. Irfan S, Zafar A, Guhar D, Ahsan T, Hasan R (2008) Metallo-beta-lactamase-producing clinical isolates of Acinetobacter species and Pseudomonas aeruginosa from intensive care unit patients of a tertiary care hospital. Indian J Med Microbiol 26: 243-245.

13. Clinical and laboratory standard institute (2006) Performance standards for antimicrobial susceptibility testing; sixteenth informational supplement. Approved standards M6A-2. Clinical and Laboratory Standards Institute, Wayne, PA, USA.

14. Gales AC, Reis AO, Jones RN (2001) Contemporary assessment of antimicrobial susceptibility testing methods for polymyxin $B$ and colistin: review of available interpretative criteria and quality control guidelines. J Clin Microbiol 39: 183-190.

15. Tan TY and Ng LS (2006) Comparison of three standardized disc susceptibility testing methods for colistin. J Antimicrob Chemother 58: 864-867.

16. Mirza IA, Hussain A, Abbasi SA, Malik N, Satti L, Farwa U (2011) Ambu Bag as a Source of Acinetobacter baumannii Outbreak in an Intensive Care Unit. J Coll Physicians Surg Pak 21: 176-178.

17. Krol V, Hamid NS, Cunha BA (2009) Neurosurgically related nosocomial Acinetobacter baumannii meningitis: report of two cases and literature review. J Hosp Infect 71: 176-180.

18. Dalgic N, Ceylan Y, Sancar M, Telhan L, Kafadar I, Cavusoglu H, Ceylan O, Hasim O (2009) Successful treatment of multidrug-resistant Acinetobacter baumannii ventriculitis with intravenous and intraventricular colistin. Ann Trop Paediatr 29: 141-147.

19. Michalopoulos A and Falagas ME (2008) Colistin and polymyxin B in critical care. Crit Care Clin 24: 377-391.

20. Oliveira MS, Prado GV, Costa SF, Grinbaum RS, Levin AS (2009) Polymyxin B and colistimethate are comparable as to efficacy and renal toxicity. Diagn Microbiol Infect Dis 65: 431-434.

21. Kuo LC, Teng LJ, Yu CJ, Ho SW, Hsueh PR (2004) Dissemination of a clone of unusual phenotype of pandrugresistant Acinetobacter baumannii at a university hospital in Taiwan. J Clin Microbiol 42: 1759-1763.

22. Ko KS, Suh JY, Kwon KT, Jung SI, Park KH, Kang CI, Chung DR, Peck KR, Song JH (2007) High rates of resistance to colistin and polymyxin $\mathrm{B}$ in subgroups of Acinetobacter baumannii isolates from Korea. J Antimicrob Chemother 60: 1163-1167.

23. Reis AO, Luz DA, Tognim MC, Sader HS, Gales AC (2003) Polymyxin-resistant Acinetobacter spp. isolates: what is next? Emerg Infect Dis 9: 1025-1027.

24. Ozdemir H, Tapisiz A, Ciftci E, Ince E, Mokhtari H, Guriz H, Aysev AD, Dogru U (2010) Successful treatment of three children with post-neurosurgical multidrug-resistant Acinetobacter baumannii meningitis. Infection 38: 241-244.

25. Falagas ME, Bliziotis IA, Tam VH (2007) Intraventricular or intrathecal use of polymyxins in patients with Gramnegative meningitis: a systematic review of the available evidence. Int J Antimicrob Agents 29: 9-25.

26. Jimenez-Mejias ME, Pichardo-Guerrero C, Marquez-Rivas FJ, Martin-Lozano D, Prados T, Pachon J (2002) Cerebrospinal fluid penetration and pharmacokinetic/pharmacodynamic parameters of intravenously administered colistin in a case of multidrug- 
resistant Acinetobacter baumannii meningitis. Eur J Clin Microbiol Infect Dis 21: 212-214.

27. Maragakis LL, Perl TM (2008) Acinetobacter baumannii: epidemiology, antimicrobial resistance, and treatment options. Clin Infect Dis 460: 1254-1263.

28. Rodriguez Guardado A BA, Asensi V, Perez F, Rial JC, Pintado V, Bustillo E, Lantero M, Tenza E, Alvarez M, Maradona JA, Carton JA (2008) Multidrug-resistant Acinetobacter meningitis in neurosurgical patients with intraventricular catheters: assessment of different treatments. J Antimicrob Chemother 61: 908-913.

29. Fulnecky EJ, Wright D, Scheld WM, Kanawati L, Shoham S (2005) Amikacin and colistin for treatment of Acinetobacter baumannii meningitis. J Infect. 51: e249-251.
30. Gilbert VE, Beals JD Jr., Natelson SE, Tyler WA (1986) Treatment of cerebrospinal fluid leaks and gram-negative bacillary meningitis with large doses of intrathecal amikacin and systemic antibiotics. Neurosurgery 18: 402-406.

\section{Corresponding author}

Ali Faisal Saleem

Department of Pediatrics and Child Health

Aga Khan University Hospital

Karachi, Pakistan

Telephone: +92-021-34864955

Fax: +92-021-34864294

Email: ali.saleem@aku.edu

Conflict of interests: No conflict of interests is declared. 